\title{
Advanced glycation endproducts interfere with integrin-mediated osteoblastic attachment to a type-I collagen matrix
}

\author{
Antonio Desmond McCarthy ${ }^{\mathrm{a}}$, Toshimasa Uemura ${ }^{\mathrm{b}}$, \\ Susana Beatriz Etcheverry ${ }^{\mathrm{a}}$, Ana María Cortizo ${ }^{\mathrm{a}, *}$ \\ a Cátedra de Bioquímica Patológica, Facultad de Ciencias Exactas, Universidad Nacional de La Plata, 47 y 115, (1900) La Plata, Argentina \\ b Age Dimension Research Center, AIST (National Institute of Advanced Industrial Science and Technology), \\ Tsukuba Central-4, 1-1-1 Higashi, Tsukuba Ibaraki, 305-8562, Japan
}

Received 13 May 2003; received in revised form 13 May 2003; accepted 29 September 2003

\begin{abstract}
The adhesion of osteoblasts to bone extracellular matrix, of which type-I collagen constitutes $>85 \%$, can modulate diverse aspects of their physiology such as growth, differentiation and mineralisation. In this study we examined the adhesion of UMR106 rat osteoblast-like cells either to a control (Col) or advanced-glycation-endproduct-modified (AGEs-Col) type I collagen matrix. We investigated the possible role of different integrin receptors in osteoblastic adhesion, by co-incubating these cells either with $\beta$-peptide (conserved sequence 113-125 of the $\beta$ subunit of integrins) or with two other peptides, RGD (Arg-Gly-Asp) and DGEA (Asp-Gly-Glu-Ala), which are recognition sequences for the $\alpha$-subunits of $\alpha_{1,5} \beta_{1}$ and $\alpha_{2} \beta_{1}$ integrins. Collagen glycation inhibited the adhesion of UMR106 osteoblasts to the matrix (40\% reduction versus Col, $P<0.001)$. $\beta$-Peptide showed a dose- and glycation-dependent inhibitory effect on adhesion, and at a concentration of $100 \mu \mathrm{M}$ decreased the attachment of UMR106 cells to both matrices (42\% to Col, $P<0.001$; and $25 \%$ to AGEs-Col, $P<0.01)$. The synthetic peptides RGD $(1 \mathrm{mM})$ and DGEA $(5 \mathrm{mM})$ inhibited the attachment of UMR106 cells to Col $(30$ and $20 \%, P<0.01$ and $P<0.001$, respectively), but not to AGEs-Col. $\beta$-Peptide induced an increase in UMR106 cell clumping and a decrease in cellular spreading, while DGEA increased spreading with cellular extensions in multiple directions. These results indicate that both $\alpha$ and $\beta$ integrin subunits participate in osteoblastic attachment to type-I collagen, probably through the $\alpha_{1,5} \beta_{1}$ and $\alpha_{2} \beta_{1}$ integrins. AGEs-modification of type-I collagen impairs the integrin-mediated adhesion of osteoblastic cells to the matrix, and could thus contribute to the pathogenesis of diabetic osteopenia.
\end{abstract}

(C) 2003 Elsevier Ltd. All rights reserved.

Keywords: Advanced glycation endproducts; Osteoblast; Adhesion; Integrin receptors; Type-I collagen

\footnotetext{
Abbreviations: AGEs, advanced glycation endproducts; ECM, extracellular matrix; RGD, Arg-Gly-Asp; DGEA, Asp-Gly-GluAla; DMEM, Dulbecco's modified Eagle's medium; EDTA, ethylenediamino-tetra-acetic acid; FBS, fetal bovine serum; PBS, phosphate buffered saline solution

* Corresponding author. Tel.: +54-221-423-5333x49; fax: +54-221-422-3409.

E-mail address: cortizo@biol.unlp.edu.ar (A.M. Cortizo).
}

\section{Introduction}

The processes of bone formation and bone turnover depend on an adequate recruitment, growth and development of osteoblastic cells (Schenk, Felix, \& Hofstetter, 1993). In turn, the attachment and spreading of these cells to an extracellular matrix or ECM (first steps in osteoblastic progression), are dependent on the interaction between various cellular adhesion 
molecules and their consensus sequences present in ECM proteins (Ducy, Schinke, \& Karsenty, 2000). Pre-eminent among these adhesion molecules are the integrin receptors or integrins.

To date, multiple integrin receptors have been described in osteoblasts. Integrins, by interaction of their $\alpha$ and $\beta$ subunits with certain consensus sequences present in ECM proteins, can aid in osteoblastic adhesion and spreading, as well as in intracellular signalling. The consensus sequences for binding of $\alpha$ subunits have been well characterised. Thus the $\alpha$ subunit of $\alpha_{2} \beta_{1}$, considered to be the main osteoblastic receptor for type-I collagen (which in turn constitutes $>85 \%$ of bone ECM), recognises the tetrapeptide sequence DGEA (Asp-Gly-Glu-Ala) present in this collagenous molecule (Takeuchi, Nakayama, \& Matsumoto, 1996). On the other hand, the $\alpha$ subunits of integrins $\alpha_{1} \beta_{1}$ and $\alpha_{5} \beta_{1}$ principally recognise the tripeptide sequence RGD (Arg-Gly-Asp), which is present in several ECM proteins such as type-I collagen, fibronectin, vitronectin and osteopontin (Ruoslahti, 1991). However, the consensus sequences for binding of $\beta$ subunits to ECM have proved to be more difficult to define. Recently, an ingenious approach to bypass this particular problem was developed by Dr. Uemura and associates (Yabe, Nemoto, \& Uemura, 1997; Uemura et al., 1997; Liu, Nemoto, Feng, \& Uemera, 1997; Sun et al., 2000; Uemura, Nemoto, \& Liu, 2000). Since all integrin $\beta$ subunits are almost identical with respect to their amino acid sequences, these investigators designed and synthesised the polypeptide Asp-Leu-Tyr-TyrLeu-Met-Asp-Leu-Ser-Tyr-Ser-Met-Lys (referred to as the $\beta$-peptide), which corresponds to the conserved amino acid sequence $113-125$ of the $\beta$ subunits of integrins (Fig. 1). The $\beta$-peptide was found to inhibit the binding of cellular integrins to ECM proteins, presumably due to competition for the same consensus sequence(s). In addition, biotinylated $\beta$-peptide was able to bind efficiently to immobilised fibronectin, fibrinogen, vitronectin or type-I collagen, with varying degrees of affinity (Liu et al., 1997).

In conditions associated with chronic hyperglycaemia, hyperlipidaemia, and/or oxidative stress (such as ageing, Alzheimer's disease, uraemia and Diabetes mellitus), there is an increase in the steady-state levels of reactive dicarbonylic compounds or "carbonyl stress", leading to an accumulation of advanced glycation-end products (AGEs) on long-lived proteins (Vlassara, Bucala, \& Striker, 1994; Baynes \& Thorpe,

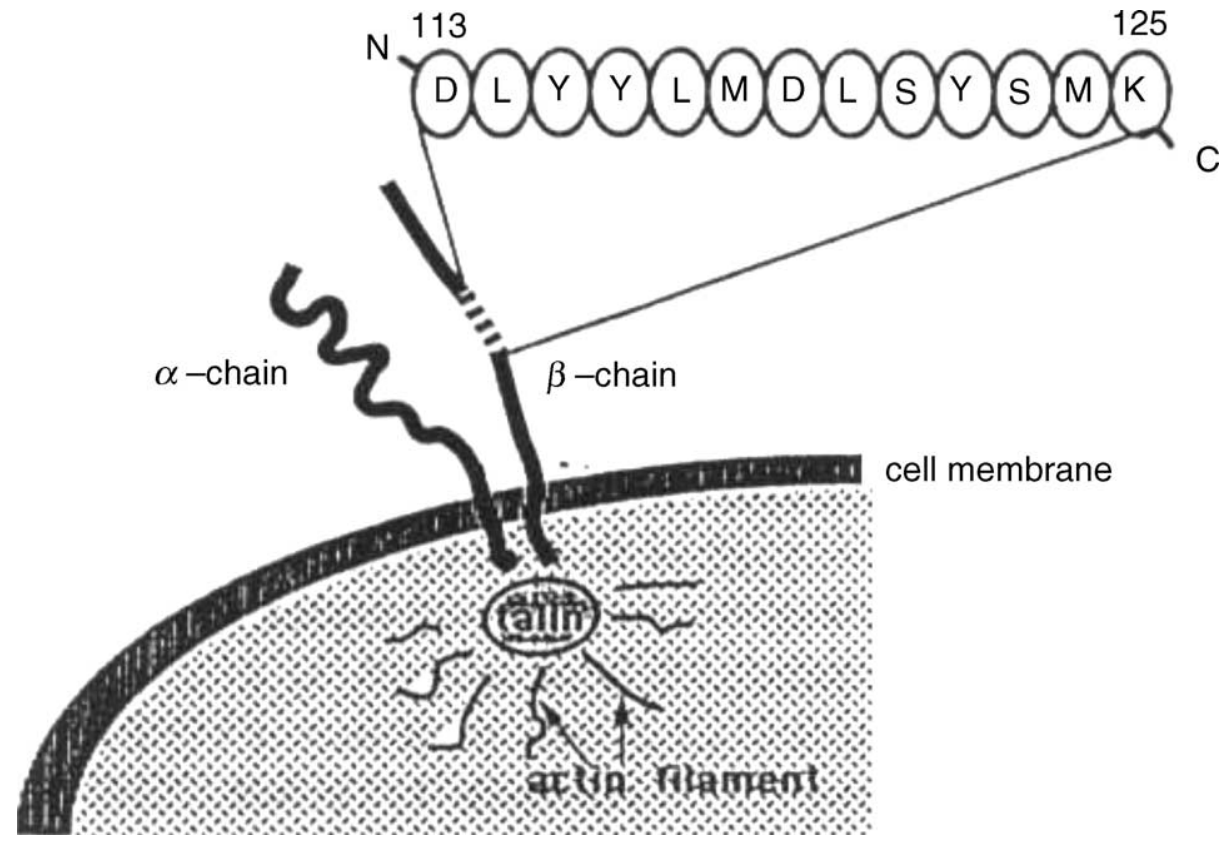

Fig. 1. Schematic structure of an integrin receptor and the $\beta$-peptide. 
1999). In particular, this accumulation of AGEs is postulated to be involved in the pathogenesis of diabetic chronic complications including osteopenia (Vlassara, 1997; McCarthy, 2000), which has recently been attributed to a decrease in osteoblast recruitment and bone-forming capacity (Krakauer et al., 1995).

Our group and other investigators have shown that AGEs-modified proteins can exert direct effects on osteoblast-like cells in culture (Miyata, Kawai, Taketomi, \& Sprague, 1996; Katayama, Akatsu, Yamamoto, Kugai, \& Nagata, 1996; Takagi et al., 1997; Paul \& Bailey, 1999; McCarthy, Etcheverry, Bruzzone, \& Cortizo, 1997; McCarthy, Etcheverry, \& Cortizo, 1999; McCarthy, Etcheverry, Bruzzone et al., 2001; McCarthy, Etcheverry, Cortizo et al., 2001; Cortizo et al., 2003). In particular, we have found that AGEs can depress osteoblastic function at several stages of their development. Thus, AGEs-modification decreases the attachment of osteoblastic UMR106 cells to a type-I collagen matrix; and long-term exposure to AGEs depresses: (a) the proliferation and differentiation of UMR106 and MC3T3E1 osteoblasts, and (b) the formation of nodules of mineralisation by MC3T3E1 cells. These effects on osteoblasts could be occurring as a consequence of an interaction between AGEs structures and their specific receptors, which we have recently described in osteoblasts (McCarthy et al., 1999; Cortizo et al., 2003). However, they could also be a consequence of an impaired interaction between other osteoblastic receptors and their AGEs-modified physiological extracellular ligands.

The object of this study was to investigate whether formation of AGEs on type-I collagen impairs its interaction with UMR106 osteoblast integrin receptors, thereby providing a possible explanation for the decreased attachment of osteoblastic UMR106 cells to an AGEs-modified type-I collagen matrix which we have previously described (McCarthy, Etcheverry, Bruzzone et al., 2001).

\section{Materials and methods}

\subsection{Materials}

Ribose, rat tail acid-soluble type I collagen, RGD peptide and DGEA peptide were purchased from Sigma (St. Louis, MO, USA). The $\beta$-peptide was synthesised by the Peptide Institute in Osaka, Japan; the purity of this synthetic product was calculated to be $95.1 \%$ by HPLC analysis (Liu et al., 1997). Dulbecco's modified Eagle's medium (DMEM), trypsine-EDTA and fetal bovine serum (FBS) were obtained from Gibco (Life Technology, Argentina). Tissue culture disposable material was from Nunc. All other chemicals and reagents were obtained from commercial sources and were of analytical grade.

\subsection{Preparation of control and AGEs-modified type-I collagen matrices}

Type I collagen was solubilized in sterile $0.02 \mathrm{~N}$ acetic acid $(2.5 \mathrm{mg} / \mathrm{ml})(\mathrm{pH} 3.0)$, poured into plastic dishes $\left(50 \mu \mathrm{g} / \mathrm{cm}^{2}\right)$ and incubated overnight at $37^{\circ} \mathrm{C}$. Collagen formed a thin film during incubation (Katayama et al., 1996). The film was washed with phosphate-buffered saline (PBS) and further incubated in PBS with or without $100 \mathrm{mM}$ ribose at $37^{\circ} \mathrm{C}$ for 3 weeks in sterile conditions (McCarthy, Etcheverry, Bruzzone et al., 2001). Finally, the plates were washed thoroughly with DMEM and the cells were plated for different experiments. AGEs-formation on collagen was monitored by measuring AGEs-specific fluorescence emission $(356 \mathrm{~nm}$ excitation wavelength and $440 \mathrm{~nm}$ emission wavelength), as we have previously described (McCarthy, Etcheverry, Bruzzone et al., 2001). After incubating 3 weeks with ribose, collagen contained more AGEs-associated fluorescence than control collagen incubated with PBS alone (30 versus 7.5 relative fluorescence units $/ \mathrm{mg}$ protein, for AGEs-modified and control collagen, respectively). Other authors have demonstrated that prolonged in vitro incubation of collagen with ribose leads to the formation of AGEs, particularly arginine-derived imidazolones (Paul, Avery, Slatter, Sims, \& Bailey, 1998).

\subsection{Measurement of free amino and guanidinium groups on collagen matrices}

The content of potentially reactive free amino groups (mainly belonging to lysine residues) on control and AGEs-modified type-I collagen was estimated with tri-nitro-benzene-sulphonic acid (Lavy, Brook, Dankner, Amotz, \& Aviram, 1991). The content of free guanidinium groups (corresponding to unchanged arginine residues) on control and AGEs-modified 
type-I collagen was estimated by the thymol/hypobromite method (Sastry \& Tummuru, 1984).

\subsection{Cell culture}

UMR106 rat osteosarcoma-derived cells were grown in $75 \mathrm{~cm}^{2}$ plastic flasks at $37^{\circ} \mathrm{C}$ in a humidified $5 \% \mathrm{CO}_{2}$ atmosphere in DMEM supplemented with $10 \%$ FBS, $100 \mathrm{U} / \mathrm{ml}$ penicillin and $100 \mathrm{mg} / \mathrm{l}$ streptomycin. This cell line has been shown to conserve certain characteristics of differentiated osteoblastic phenotype (Partridge, Alcorn, Michelangeli, Ryan, \& Martin, 1983). After 5-7 days, cells were sub-cultured using trypsin/EDTA and replated on plastic, on control collagen or on glycated collagen to begin the experiments.

\subsection{Cell adhesion and spreading: effect of various peptides}

UMR106 osteoblast-like cells were plated in DMEM-10\% FBS on plastic, or on control or AGEs-modified type-I collagen-coated 24 well plates, at a seeding density of $2.5 \times 10^{5}$ cells $/ \mathrm{ml}$, in the presence or absence of different peptides: RGD $(1 \mathrm{mM})$, DGEA $(5 \mathrm{mM})$, or $\beta$-peptide $(50$ or $100 \mu \mathrm{M})$. Cells were allowed to adhere for $1 \mathrm{~h}$ at $37^{\circ} \mathrm{C}$. Each well was then washed with PBS, fixed with methanol for $5 \mathrm{~min}$ and stained with Giemsa for $10 \mathrm{~min}$. Cells adhered to plastic or collagen matrices were evaluated microscopically by counting several representative fields per well. We have previously found that a linear regression of the adhesion assay occurs between $1 \times 10^{5}$ and $1 \times 10^{6}$ cells $/ \mathrm{ml}(y=0.826+0.759 x$, $r=0.993$ ) (McCarthy, Etcheverry, Bruzzone et al., 2001). Cellular spreading was assessed by the observation of stained cells that presented more than one corner, in several representative microscopic fields per well.

\subsection{Statistical analysis}

At least three independent experiments were run for each experimental condition. Results are expressed as mean \pm S.E.M. Statistical analysis of the data was performed by Student's $t$-test.

\section{Results}

\subsection{Content of free lysine and arginine residues on control and glycated collagen matrices}

In the case of AGEs-modified type-I collagen, the content of potentially reactive free amino groups (mainly belonging to lysine residues) was $72 \%$ of control (non-glycated) collagen. In addition, AGEscollagen also had a lower content $(78 \%)$ of free arginine residues than control collagen.

\subsection{Osteoblastic attachment to different surfaces}

As described in Section 2, UMR106 osteoblastic cells were plated on plastic, or on control or AGEs-modified type-I collagen-coated wells. In our experimental conditions, control (non-glycated) type-I collagen was found to be a better substratum than plastic for the adhesion of UMR106 cells, increasing osteoblast attachment by $25 \%$ (Table 1). However, AGEs modification of the type-I collagen film resulted in a matrix to which the osteoblasts adhered less easily ( $40 \%$ reduction in adhesiveness versus control collagen) (Table 1).

\subsection{Effect of different peptides on osteoblast attachment to collagen matrices}

UMR106 cells were plated on control or AGEsmodified type-I collagen-coated wells, in the presence

Table 1

UMR106 osteoblasts were plated on different surfaces for $1 \mathrm{~h}$ at $37^{\circ} \mathrm{C}$, without competing peptides. Cell adhesion was evaluated microscopically as described in Section 2

\begin{tabular}{lll}
\hline Surface & Cell attachment (cells/field \pm S.E.M.) & Statistical significance \\
\hline Plastic & $69 \pm 4$ & \\
Control type-I collagen & $86 \pm 5$ & $P<0.02$ (vs. plastic) \\
AGEs-modified type-I collagen & $54 \pm 4$ & $P<0.001$ (vs. control type-I collagen) \\
\hline
\end{tabular}




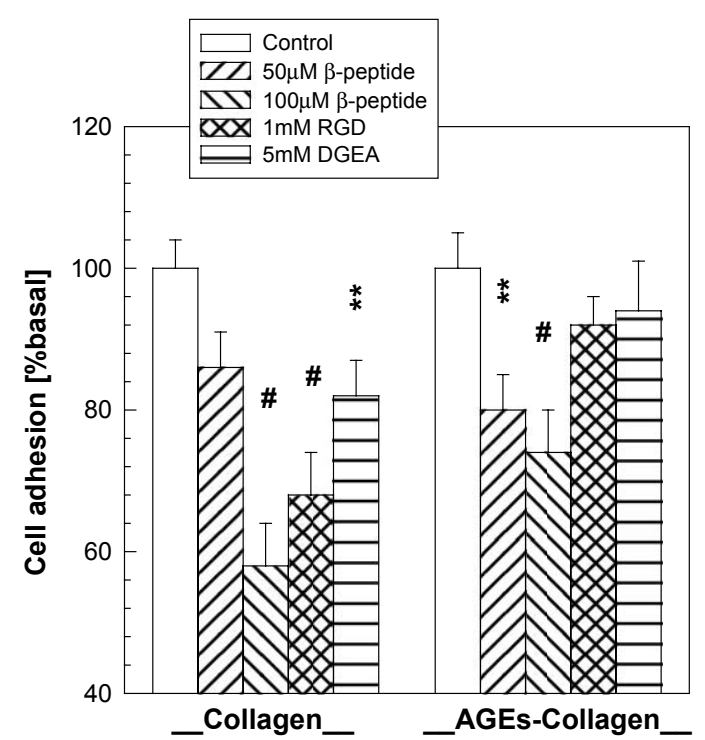

Fig. 2. Adhesion of UMR106 osteoblastic cells to a control or AGEs-modified type-I collagen matrix, in the presence or absence of $1 \mathrm{mM}$ RGD, $5 \mathrm{mM}$ DGEA or $\beta$-peptide $(50$ or $100 \mu \mathrm{M})$. Differences versus control (cells plated without peptides) are: ${ }^{* *} P<0.01 ;{ }^{\#} P<0.001$.

or absence of different peptides that interfere with integrin/ECM interaction. When the osteoblasts were plated on non-glycated collagen matrices, co-incubation with DGEA, RGD or $\beta$-peptide inhibited cellular adhesion in comparison with control (no peptide) (Fig. 2). In the case of $\beta$-peptide, this inhibition was dose-dependent and took place at molar concentrations 10-20 times lower than RGD, and 50-100 times lower than DGEA.

When UMR106 osteoblastic cells were plated on AGEs-modified type-I collagen, coincubation with RGD or DGEA had no effect on cell adhesion in comparison with control (no peptide) (Fig. 2). On the other hand, $100 \mu \mathrm{M} \beta$-peptide still retained an inhibitory effect on cell attachment, though significantly less than with non-glycated collagen (Figure 2).

\subsection{Effect of different peptides on osteoblastic spreading}

We next evaluated the possible effects on osteoblastic spreading of co-incubation with RGD, DGEA or $\beta$-peptide. When co-incubated with $\beta$ peptide, UMR106 cells plated both on glycated and non-glycated collagen matrices showed an increase in cell clumping, and a decrease in cellular spreading, in comparison with controls (no peptide) (Fig. 3). On the other hand, UMR106 osteoblasts plated on non-glycated collagen in the presence of DGEA exhibited greater spreading than the corresponding control (no peptide), with cellular extensions in multiple directions (Fig. 4). However, this effect of DGEA co-incubation on cellular spreading was not apparent when the osteoblasts were plated on AGEs-modified collagen (data not shown). Finally, RGD did not induce changes in cellular spreading in any case.

\section{Discussion}

It has been established that sustained hyperglycaemia and/or hyperlipidaemia can cause tissue damage through several mechanisms, including the accelerated formation of AGEs on both intracellular and extracellular proteins, altering their bioactivity and associative properties. In particular, the integrity of the extracellular matrix (ECM) is essential to maintain the normal function of osteoblasts, the main cell type involved in the formation of bone $(\mathrm{Ng}$, Romas, Donnan, \& Findlay, 1997). The accumulation of AGEs on bone type-I collagen has been previously described in both diabetes and ageing (Locatto et al., 1993; Tomasek, Meyers, Basinger, Green, \& Shew, 1994; Katayama et al., 1996). Thus, an AGEs-modified ECM may have important consequences on the normal bone-forming activity of osteoblasts, possibly through changes in cell-matrix interactions.

Osteoblasts are known to possess AGEs-specific cell surface receptors (McCarthy et al., 1999; Cortizo et al., 2003) whose affinity for AGEs moieties would be expected to induce an increase in the attachment of these cells to an AGEs-enriched collagen substratum. However, in the light of our present and previous (McCarthy, Etcheverry, Bruzzone et al., 2001) studies, it is evident that UMR106 osteoblastic cells adhere less easily to a type-I collagen matrix when it is modified by AGEs. Thus, we hypothesised that this decreased attachment might be the consequence of an impaired interaction between other osteoblastic receptors (such as integrins) and AGEs-modified type-I collagen.

To test this hypothesis we employed two kinds of peptides, which we co-incubated with the osteoblasts 
(A)

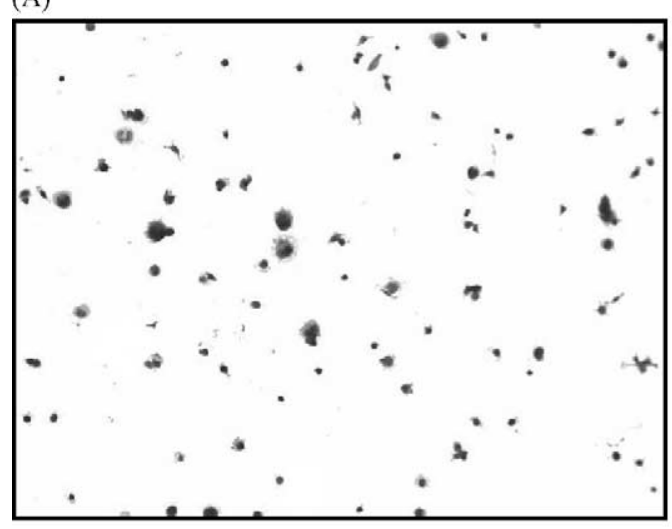

(C)

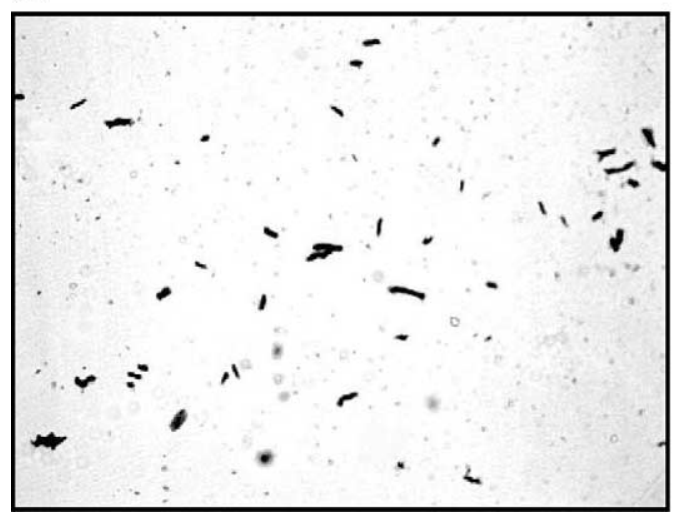

(B)

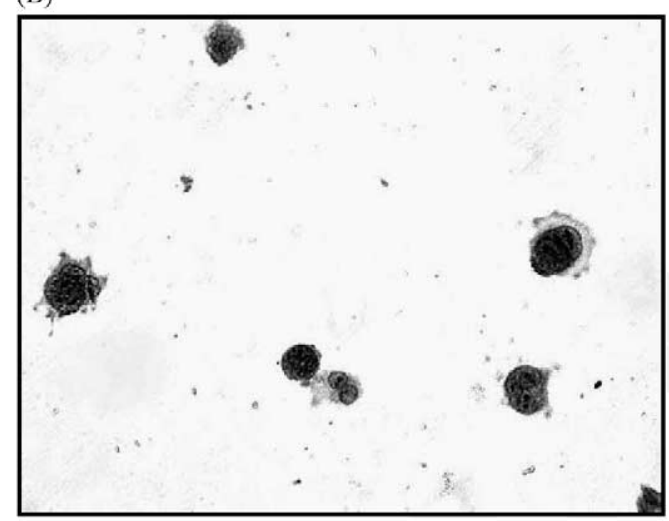

(D)

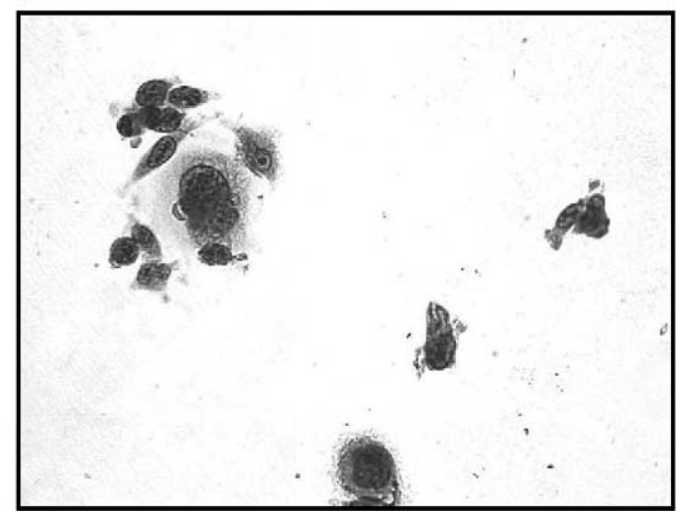

Fig. 3. Effect of co-incubation with $100 \mu \mathrm{M} \beta$-peptide (C and D) on the morphological features of adhering UMR106 osteoblastic cells, compared to control (no peptide, cells plated on type-I collagen) (A and B). Objective: $10 \times$ (A and C); $40 \times(B$ and D).

in adhesion experiments: (a) RGD and DGEA, which mimic the consensus sequences of ECM proteins for the $\alpha$ subunits of $\alpha_{1,5} \beta_{1}$ and $\alpha_{2} \beta_{1}$ integrin receptors, respectively, and therefore interfere with cell adhesion by binding to these integrins; and (b) $\beta$-peptide, a 13-aminoacid conserved sequence of the $\beta$ subunits of integrins (Fig. 1), which can inhibit cell attachment by binding to the corresponding consensus sequences of ECM proteins. As expected, DGEA inhibited osteoblastic attachment to non-glycated type-I collagen by about $20 \%$ (Fig. 2). Surprisingly however, RGD also inhibited osteoblastic attachment to this control matrix by about $30 \%$ (Fig. 2). This latter result differs to reports by other authors (who found no effect of RGD on osteoblastic adhesion to non-glycated type-I collagen) (Takeuchi, Nakayama, \& Matsumoto, 1996), but is in agreement with the presence of RGD in type-I collagen. Certain experimental conditions could expose this RGD sequence for interaction with $\alpha_{1,5} \beta_{1}$ integrins present on osteoblasts, while different conditions (such as employed by other authors) may change type-I collagen conformation in a way which would impede interaction due to steric hindrance. We next used RGD, DGEA or $\beta$-peptide to try to block the adhesion of UMR106 osteoblasts to an AGEs-modified type-I collagen matrix. As a result, we found that while $\beta$-peptide still retained roughly about half of its inhibitory potential (compared to control collagen), the inhibitory effect of RGD and DGEA on osteoblastic attachment was completely abolished.

In general these results are in agreement with our hypothesis that AGEs-modification of type-I collagen, interferes with the adhesion of osteoblasts to this ECM protein mediated by the $\alpha$ and $\beta$ subunits of integrin 


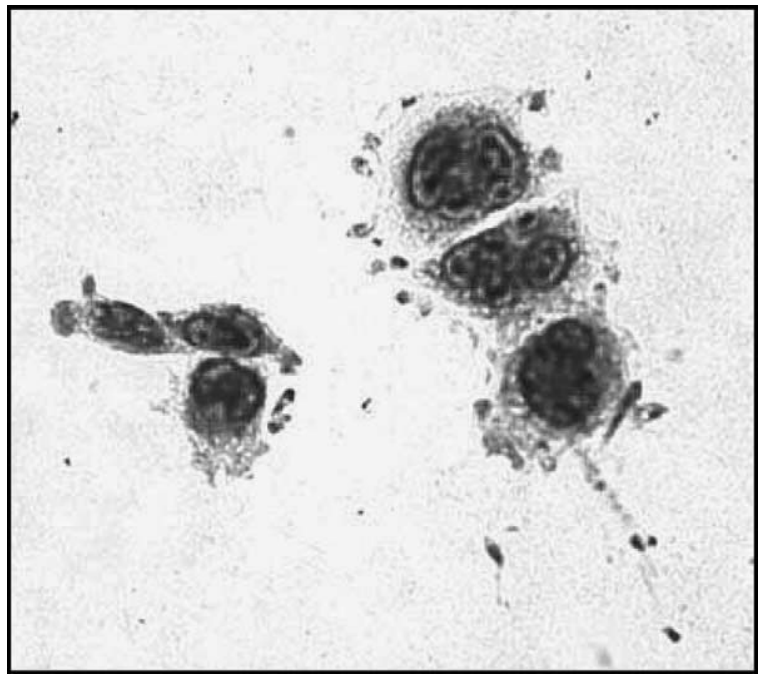

Fig. 4. Effect of co-incubation with $5 \mathrm{mM}$ DGEA on the morphological features of adhering UMR106 osteoblastic cells. Note the difference with control in Fig. 3B. Objective: $40 \times$.

receptors. In this context, it is significant to note that the proportion of cell attachment to control collagen which can be attributed to integrin/ECM interaction in our experiments (20-40\%) (Fig. 1), is practically coincident with the AGEs-induced decrease in osteoblast adhesion to glycated collagen observed in this (Table 1) and previous studies (40\%) (McCarthy, Etcheverry, Bruzzone et al., 2001).

There are several mechanisms by which AGEs structures could impede integrin-mediated UMR106 cell adhesion. In the first place, our present results indicate that glycated type-I collagen (versus control collagen) shows a four-fold increase in AGEs-associated fluorescence, a $28 \%$ reduction in free lysine $\varepsilon$-amino residues and a $22 \%$ reduction in free arginine guanidinium residues. These in vitro results are similar to the in vivo results found by other authors. For instance, Tomasek et al. demonstrated that in 30-month old (ageing) nondiabetic rats, cortical bone collagen AGEs-specific fluorescence was 2.03 times higher that in young (3-month old) non-diabetic rats (Tomasek et al., 1994). These investigators also found a 1.7-fold further increase in bone collagen fluorescence, in rats that had been rendered diabetic for 2 months by streptozotocin treatment. Other studies with non-diabetic human individuals have found an age-induced decrease in free lysine and arginine residues of articular cartilage collagen, this decrease reaching $7-10 \%$ of lysine/arginine residues over the entire human life span ( $0-80$ years) (Verzijl et al., 2000). Although there are no reports in the literature indicating the levels of AGEs in type-I collagen from bone of human individuals with long-standing Diabetes mellitus, it is not inconceivable to suppose that such levels could lead to an approximately $15-20 \%$ decrease in collagen free lysine and arginine residues (that is, approximately double that of ageing non-diabetic humans). Interestingly, it has been estimated that a 2-week incubation of collagen with $0.1 \mathrm{M}$ ribose is equivalent to the exposure of this protein to physiological glucose concentrations for 20 years (Tanaka, Avigad, Eikenberry, $\&$ Brodsky, 1988). Thus, our in vitro glycated type-I collagen film could be an adequate model to represent the AGEs-modified bone collagen of individuals with long-standing Diabetes mellitus.

Arginine is included in the RGD tripeptide consensus sequence for $\alpha_{1,5} \beta_{1}$ integrins, and so its irreversible modification by a voluminous AGEs moiety would be expected to directly interfere with $\alpha_{1,5} \beta_{1}$ integrin-mediated osteoblastic adhesion. In fact, our present results are very similar to reports by other authors (Sakata et al., 2000), who have found a decrease in the attachment of vascular smooth muscle cells to AGEs-modified fibronectin and type-I collagen (versus control proteins). These authors also noted that AGEs-modification of these ECM proteins decreased their content of free arginine residues, and abolished the inhibitory effect of externally added RGD on vascular smooth muscle cell adhesion to the matrix.

AGEs have also been shown to modify the structure and bioactivity of the proteins on which they are formed due to their intrinsic volume and chemical reactivity, and because they can form intra- and intermolecular crosslinks (Vlassara et al., 1994; Vlassara, 1997; McCarthy, Cortizo, Giménez Segura, Bruzzone, \& Etcheverry, 1998; Baynes \& Thorpe, 1999; McCarthy, 2000). Thus, the accumulation of AGEs on a type-I collagen matrix can induce important conformational changes in its structure, which would impair its ability to interact efficiently with cellular receptors such as integrins. In fact, this is probably the mechanism which most adequately accounts for the relative or absolute inability of DGEA or $\beta$-peptide to inhibit osteoblastic adhesion to a glycated collagen matrix. 
In the present study, we also evaluated the possible effect of DGEA, RGD or $\beta$-peptide on osteoblastic spreading. Although in our experimental conditions RGD did not induce changes in cellular spreading, we found that in the presence of $\beta$-peptide UMR106 cells showed an increase in cell clumping and a decrease in cellular spreading, when plated both on glycated and non-glycated collagen matrices (Fig. 3). In the presence of DGEA, UMR106 osteoblasts plated on non-glycated collagen (but not on AGEs-modified collagen) showed an increase in spreading, with cellular extensions in multiple directions (Fig. 4). All in all, these morphological features are in general agreement with the results of adhesion experiments, and suggest that osteoblastic cells might be particularly susceptible to alterations in cell-cell and cell-matrix interactions when in the presence of molecules which compete with integrin binding to ECM proteins. One of the authors of the present work (T. Uemura) has previously examined the effects of $\beta$-peptide on osteoblasts, fibroblasts and hepatoma cells cultured on different ECM proteins (Yabe, Nemoto, \& Uemura, 1997; Uemura et al., 1997; Liu et al., 1997; Sun et al., 2000; Uemura et al., 2000). All the data from these past experiments have clearly shown a dose-dependent inhibitory effect of $\beta$-peptide on cell adhesion, via interference with integrin recognition of ECM protein consensus sequences. Additionally, in the experiments with osteoblasts and hepatoma cells, $\beta$-peptide induced a decrease in cellular spreading and an increase in cellular aggregation similar to that which we have described in the present study. In particular, this cellular aggregation (clumping) could be due either to an enhancement of cell-cell interaction induced directly by $\beta$-peptide, or more probably to an increase in cellular self-aggregation secondary to their detachment from the matrix by $\beta$-peptide.

In conclusion, our present experiments confirm the result of previous studies (McCarthy, Etcheverry, Bruzzone et al., 2001) showing that glycation of a type-I collagen matrix decreases its capacity for the adhesion of UMR106 osteoblast-like cells. In addition, we have provided evidence which strongly suggests that this decreased attachment is the consequence of an impaired interaction between osteoblastic $\alpha_{1,5} \beta_{1}$ and $\alpha_{2} \beta_{1}$ integrin receptors and AGEs-modified type-I collagen, due to a direct or indirect structural modification of the consensus sequences for these integrins. If these results were to be extrapolated to a clinical setting, AGEs-enriched bone ECM proteins would possess a decreased potential for osteoblastic attachment (and recruitment), and could thus contribute to the development of diabetic osteopenia.

\section{Acknowledgements}

This study was partially supported by grants from Ministerio de Salud de la Nación Argentina (Beca Ramón Carrillo-Arturo Oñativia 2001), CONICET (PIP 1044/98) and Universidad Nacional de La Plata. AMC is a member of the Carrera del Investigador, CICPBA, and SBE is a member of the Carrera del Investigador, CONICET. The authors are indebted to Dr. Adriana Scoccia for the determination of lysine and arginine unmodified residues.

\section{References}

Baynes, J. W., \& Thorpe, S. R. (1999). Role of oxidative stress in diabetic complications: a new perspective on an old paradigm. Diabetes, 48, 1-9.

Cortizo, A. M., Lettieri, M. G., Barrio, D. A., Mercer, N., Etcheverry, S. B., \& McCarthy, A. D. (2003). Advanced glycation endproducts (AGEs) induce concerted changes in the osteoblastic expression of their receptor RAGE and in the activation of extracellular signal-regulated kinases (ERK). Molecular and Cellular Biochemistry, 250, 1-10.

Ducy, P., Schinke, T., \& Karsenty, G. (2000). The osteoblast: a sophisticated fibroblast under central surveillance. Science, 289, 1501-1504.

Katayama, Y., Akatsu, T., Yamamoto, M., Kugai, N., \& Nagata, N. (1996). Role of nonenzymatic glycosylation of type I collagen in diabetic osteopenia. Journal of Bone and Mineral Research, 11, 931-937.

Krakauer, J. C., McKenna, M. J., Buderer, N. F., Rao, D. S., Whitehouse, F. W., \& Parfitt, A. M. (1995). Bone loss and bone turnover in diabetes mellitus. Diabetes, 44, 775782.

Lavy, A., Brook, G. J., Dankner, G., Amotz, A. B., \& Aviram, M. (1991). Enhanced in vitro oxidation of plasma lipoproteins derived from hypercholesterolemic patients. Metabolism, 40, 794-799.

Liu, Y. K., Nemoto, A., Feng, Y., \& Uemura, T. (1997). The binding ability to matrix proteins and the inhibitory effects on cell adhesion of synthetic peptides derived from a conserved sequence of integrins. Journal of Biochemistry (Tokyo), 121, 961-968.

Locatto, M. E., Abrazon, H., Caferra, D., Fernández, M. C., Alloatti, R., \& Puche, R. C. (1993). Growth and development of 
bone mass in untreated alloxan diabetic rats. Effects of collagen glycosylation and parathyroid activity on bone turnover. Bone \& Mineral, 23, 129-144.

McCarthy, A. D. (2000). Glycation, glycoxidation and carbonyl stress: role in the vascular complications of Diabetes mellitus. Revista Argentina de Endocrinología y Metabolismo, 37, 141163.

McCarthy, A. D., Cortizo, A. M., Giménez Segura, G., Bruzzone, L., \& Etcheverry, S. B. (1998). Non-enzymatic glycosylation of alkaline phosphatase alters its biological properties. Molecular and Cellular Biochemistry, 181, 63-69.

McCarthy, A. D., Etcheverry, S. B., Bruzzone, L., Lettieri, M. G., Barrio, D. A., \& Cortizo, A. M. (2001a). Non-enzymatic glycosylation of a type I collagen matrix: effects on osteoblastic development and oxidative stress. BioMedCentral Cell Biology, 2,16 .

McCarthy, A. D., Etcheverry, S. B., \& Cortizo, A. M. (2001b). Effect of advanced glycation endproducts on the secretion of insulin-like growth factor-I and its binding proteins: role in osteoblast development. Acta Diabetologica, 38, 113-122.

McCarthy, A. D., Etcheverry, S. B., Bruzzone, L., \& Cortizo, A. M. (1997). Effects of advanced glycation end-products on the proliferation and differentiation of osteoblast-like cells. Molecular and Cellular Biochemistry, 170, 43-51.

McCarthy, A. D., Etcheverry, S. B., \& Cortizo, A. M. (1999). Advanced glycation end product specific receptors in rat and mouse osteoblast-like cells: regulation with stages of differentiation. Acta Diabetologica, 36, 45-52.

Miyata, T., Kawai, R., Taketomi, S., \& Sprague, S. M. (1996). Possible involvement of advanced glycation end-products in bone resorption. Nephrology Dialysis Transplantation, 11, 5457.

Ng, K. W., Romas, E., Donnan, L., \& Findlay, D. M. (1997). Bone biology. Baillères Clinical Endocrinology and Metabolism, 11, $1-21$.

Partridge, N. C., Alcorn, D., Michelangeli, V. P., Ryan, G., \& Martin, T. J. (1983). Morphological and biochemical characterization of four clonal osteogenic sarcoma cell lines of rat origin. Cancer Research, 43, 4308-4312.

Paul, R. G., Avery, N. C., Slatter, D. A., Sims, T. J., \& Bailey, A. J. (1998). Isolation and characterisation of advanced glycation end-products derived from the in vitro reaction of ribose and collagen. Biochemical Journal, 330, 1241-1248.

Paul, R. G., \& Bailey, A. J. (1999). The effect of advanced glycation end-product formation upon cell-matrix interactions. International Journal of Biochemistry and Cell Biology, 31, 653-660.

Ruoslahti, E. (1991). Integrins. Journal of Clinical Investigation, $87,1-5$.

Sakata, N., Sasatomi, Y., Meng, J., Ando, S., Uesugi, N., Takebayashi, S., Nagai, R., \& Horiuchi, S. (2000). Possible involvement of altered RGD sequence in reduced adhesive and spreading activities of advanced glycation end product-modified fibronectin to vascular smooth muscle cells. Connective Tissue Research, 41, 213-228.

Sastry, C. S. P., \& Tummuru, M. K. (1984). Spectrophotometric determination of arginine in proteins. Food Chemistry, 15, 257260.

Schenk, R.K., Felix, R., Hofstetter, W. (1993). Morphology of connective tissue: bone. In P. M. Royce, B. Steinmann (Eds.), Connective tissue and its heritable disorders: molecular, genetic and medical aspects (1st ed., pp. 85-101). New York: Wiley.

Sun, J. J., Zhou, X. D., Liu, Y. K., Tang, Z. Y., Sun, R. X., Zhao, Y., \& Uemura, T. (2000). Inhibitory effects of synthetic beta peptide on invasion and metastasis of liver cancer. Journal of Cancer Research and Clinical Oncology, 126, 595-600.

Takagi, M., Kasayama, S., Yamamoto, T., Motomura, T., Hashimoto, K., Yamamoto, H., Sato, B., Okada, S., \& Kishimoto, T. (1997). Advanced glycation endproducts stimulate interleukin-6 production by human bone-derived cells. Journal of Bone and Mineral Research, 12, 439-446.

Takeuchi, Y., Nakayama, K., \& Matsumoto, T. (1996). Differentiaton and cell-surface expression of transforming growth factor- $\beta$ receptors are regulated by interaction with matrix collagen in murine osteoblastic cells. Journal of Biological Chemistry, 271, 3938-3944.

Tanaka, S., Avigad, G., Eikenberry, E. F., \& Brodsky, B. (1988). Isolation and partial characterization of collagen chains dimerized by sugar-derived crosslinks. Journal of Biological Chemistry, 263, 17650-17657.

Tomasek, J. J., Meyers, S. W., Basinger, J. B., Green, D. J., \& Shew, R. L. (1994). Diabetic and age-related enhancement of collagen-linked fluorescence in cortical bones of rats. Life Sciences, 55, 855-861.

Uemura, T., Liu, Y. K., Feng, Y., Nemoto, A., Yabe, T., Ushida, T., Miyamoto, H., \& Tateishi, T. (1997). The role of sialoproteins in recognition of bone surface by osteoblasts via integrin. Materials Science \& Engineering Communications, 4, 303-309.

Uemura, T., Nemoto, A., \& Liu, Y. K. (2000). Synthetic peptide derived from a conserved sequence of integrin $\mathrm{b}$ subunit. Research Advances in Bioscience \& Bioengineering, 1, 65-83.

Verzijl, N., DeGroot, J., Oldehinkel, E., Bank, R. A., Thorpe, S. R., Baynes, J. W., Bayliss, M. T., Bijlsma, J. W. J., Lafeber, F. P. J. G., \& TeKoppele, J. M. (2000). Age-related accumulation of Maillard reaction products in human articular cartilage collagen. Biochemical Journal, 350, 381-387.

Vlassara, H. (1997). Recent progress in advanced glycation endproducts and diabetic complications. Diabetes, 46(Suppl. 2), S19-S25.

Vlassara, H., Bucala, R., \& Striker, L. (1994). Pathogenic effects of advanced glycosylation: biochemical, biologic and clinical implications for diabetes and ageing. Laboratory Investigation, 70, 138-151.

Yabe, T., Nemoto, A., \& Uemura, T. (1997). Recognition of osteopontin by rat bone marrow derived osteoblastic primary cells. Bioscience Biotechnology \& Biochemistry, 61, 754-756. 